\title{
Zur Edition historischer Tonaufnahmen - ein Praxisbericht
}

https://doi.org/10.1515/bfp-2018-0001

Zusammenfassung: Auf Basis der Praxis des Phonogrammarchivs der Österreichischen Akademie der Wissenschaften präsentiert der Beitrag neben einigen generellen Überlegungen zwei Workflows $\mathrm{zu}$ inhaltlichen und technischen Aspekten einer kommentierten Edition historischer Tonaufnahmen. Thematisiert werden u. a. die quellenkritische Aufbereitung und Kontextualisierung sowie Re-recording, Digitalisierung und Signalverbesserung.

Schlüsselwörter: Historische Tonaufnahmen; Edition; Rerecording; Signalverbesserung

\section{On the Edition of Historical Sound Recordings - Reflec- tions and Workflows}

Abstract: Following the code of practice established by the Phonogrammarchiv of the Austrian Academy of Sciences, this contribution - alongside some general reflections presents two workflows on the content-related and technical aspects of an annotated edition of historical sound recordings. The topics include source-critical preparation and contextualisation as well as re-recording, digitisation, and signal processing.

Keywords: Historical sound recordings; edition; re-recording; signal processing

\section{Einleitung und Überblick}

Das Phonogrammarchiv (PhA) der Österreichischen Akademie der Wissenschaften (ÖAW), 1899 als erstes Schallarchiv der Welt gegründet, veröffentlicht seit 1999 eine Gesamtausgabe der Historischen Bestände 1899-1950 auf CD. ${ }^{1}$ Diversen Vorarbeiten für die Edition dieser unikalen, bei Feldforschungen oder im Archiv entstandenen akus-

1 Für einen ersten Überblick über die Geschichte, Aufgaben und Bestände des PhA empfiehlt sich dessen Webseite mit Online-Katalog und weiterführender Literatur unter http://www.phonogrammarchiv. at.

*Kontaktperson: Christian Liebl, Christian.Liebl@oeaw.ac.at tischen Aufnahmen auf mechanischen Tonträgern, die aus der Zeit vor der Einführung der Magnetbandtechnik im PhA (1951) stammen und im Memory of the World Register der UNESCO eingetragen sind, widmet sich die 2015 abgeschlossene Master-These ${ }^{2}$ des Autors in drei Hauptkapiteln. Sie werden untenstehend kurz dargelegt, um einen Einblick in die Gesamtkonzeption der These zu geben, wobei der Schwerpunkt dieses Beitrags aber in der Vorstellung der beiden Workflows liegt.

Probleme und Methoden der Erschließung, Kontextualisierung und Edition historischer Tondokumente bilden den Mittelpunkt des ersten Kapitels der These, an dessen Beginn Betrachtungen zum Wert historischer Tonaufnahmen stehen, bevor dann Wesen und Ziele der Gesamtausgabe sowie deren Editionsprinzipien erläutert werden. Im Anschluss folgen Überlegungen zu einer zukünftigen Publikationsform (Online/Open Access) ${ }^{3}$ sowie ein Vergleich mit der CD-Reihe des Berliner PhonogrammArchivs. Die beiden nächsten Abschnitte umfassen zwei exemplarische, praxisorientierte Workflows für die kommentierte Quellenedition historischer Tondokumente auf Basis des im PhA vorliegenden Materials - insbesondere der Aufnahme-Protokolle und der dazugehörigen Phonogramme. Der Workflow zur inhaltlichen Erschließung ist eher diskursiv angelegt und thematisiert neben wichtigen Arbeitsschritten auch prinzipielle editorische Fragen, während der technische Workflow als klassischer Leitfaden konzipiert ist.

2 Liebl (2015).

3 Bislang bestand eine Publikation im Rahmen der Gesamtausgabe in der Regel aus: Audio-CD(s), Daten-CD („data disc“ mit Scans der Aufnahme-Protokolle und PDF-Files schwer zugänglicher Sekundärliteratur, diverser Materialien sowie vertiefender Beiträge) und Booklet (Einleitung, grundlegende Texte, Transkriptionen der Aufnahmen). Ab Serie 16 werden jedoch auf der „data disc“ auch nahezu alle Texte des Booklets Platz finden, was v.a. Lesbarkeit und Layout verbessert sowie die elektronische Durchsuchbarkeit ermöglicht. Es wäre auch zu überlegen, zukünftige Serien der Gesamtausgabe zusätzlich oder ausschließlich online zu publizieren (wobei der Zugriff kostenpflichtig oder gratis - „Open Access“ - sein könnte). Dafür sprächen - abgesehen von den niedrigeren Kosten - u.a. die größere Reichweite sowie die Möglichkeiten des individuellen Downloads einzelner Audio-Files, der Verlinkung und ständigen Aktualisierung. 
Im zweiten Kapitel der These wird eine Auswahl unveröffentlichter historischer Tonaufnahmen des PhA aus den Jahren 1911 bis 1918 für die künftige Edition vorerschlossen. Abgesehen von der quellenkritischen Aufbereitung der Protokolle liegt der Schwerpunkt dabei auf den Biografien der Feldforscher (in diesem Falle Sprachwissenschaftler) und der Phonographierten sowie auf der Kontextualisierung der Tondokumente. Konkret handelt es sich um folgende kleinere Sammlungen:

a) Eugen Herzog: „Französische und deutsche Aufnahmen, Archiv 1911“/„Czernowitz 1912-13“

b) Carlo Battisti: „Italienische Dialekte, Archiv 1913“

c) Josef Balassa: „Ungarn 1914-15“

d) Karl von Ettmayer: „Italienische Kriegsgefangene 1918“/„Grödnertal 1918“

Im letzten Kapitel galt es, auf Grundlage der $\operatorname{ISAD}(G)$ Internationale Grundsätze für die archivische Verzeichnung, einen Archivbehelf für ein Konvolut bislang ungesichteter Materialien aus dem $\mathrm{PhA}$ zu erstellen, um diese Quelle (v.a. allgemeine Akten, Personalakten und Zeitungsausschnitte der Jahre 1899-1955) für Editionen und Recherchen zur Geschichte des PhA entsprechend nutzbar zu machen.

\section{Workflow I: Inhaltliche Aspekte}

\subsection{Einführung}

Trotz ihrer Defizite - wie etwa mangelnde Tonqualität oder geringe Aufnahmedauer (bei Phonogrammen maximal drei Minuten) - werden historische Tondokumente heutzutage von der Forschung im Allgemeinen durchaus wertgeschätzt, wenn auch nicht gleichermaßen in allen Disziplinen. Unabdingbare Voraussetzung ist jedenfalls eine editorische Aufbereitung in inhaltlicher und technischer Hinsicht. Eine Edition wie die Gesamtausgabe richtet sich aber auch an die Ursprungsländer der Aufnahmen, die über solch einzigartige Quellen vielfach gar nicht verfügen, und stellt somit einen Akt der Repatriierung von immateriellem Kulturgut dar. Um die Internationalität der Gesamtausgabe zu gewährleisten, ist die Publikationssprache in der Regel Englisch; im Sinne des Zielpublikums werden aber gelegentlich auch zwei- oder mehrsprachige Editionen publiziert.

\subsection{Editionsschritte}

Ausgehend von den sogenannten, im $\mathrm{PhA}$ vorliegenden Protokollen - also der zeitgenössischen schriftlichen Dokumentation zu den Aufnahmesitzungen (ansatzweise vergleichbar den deskriptiven „Metadaten“ eines „Dublin Core Metadata Element Set"), ${ }^{4}$ ohne deren Information der wissenschaftliche Nutzen akustischer Zeugnisse erheblich eingeschränkt ist - lassen sich folgende Editionsschritte definieren:

1. Inhaltliche Zusammenstellung der Aufnahmen zu Serien: In erster Linie widmet sich eine Serie einer bestimmten Region oder Thematik bzw. einem oder mehreren Forschern/Forschungsprojekten. ${ }^{5}$ Sie besteht aus einzelnen Archivnummern, aufgrund der kurzen Aufnahmedauer musste eine Darbietung jedoch oft auf mehrere Phonogramme (und somit zusammengehörige Archivnummern) verteilt werden, die zu einem CD-Track zusammenzufassen sind. Im Anschluss an die Feststellung der im Rahmen eines Projektes getätigten Feld- und/oder Archivaufnahmen ist ihre Reihenfolge in der Edition zu klären. In der Regel wird es sinnvoll sein, bei kleinen Sammlungen die chronologische, aus den Datumsangaben der Protokolle ersichtliche Abfolge in der Edition abzubilden. Bei größeren Sammlungen hingegen ist eine übergeordnete Gruppierung nach inhaltlichen Kriterien vorzuziehen, z.B. nach Genres (Sprach-/Musikaufnahmen; diese zerfallen wiederum in Vokal-/Instrumentalmusik etc.) oder nach Sprachgebieten und Dialektorten (wie bei unserer Edition der Schweizer Aufnahmen), wobei innerhalb der jeweiligen Gruppen die Aufnahmen chronologisch zusammenzufassen sind. Dies erlaubt einen besseren Überblick über den Bestand (sowie letztend-

4 Das Aufnahmeprotokoll bestand aus dem Protokollkopf, also dem Vordruck für die Angaben zur phonographierten Person und zur Aufnahme (technische und inhaltliche Daten) sowie aus dem Freitextfeld für Inhalt, Transkription und Übersetzung. Diese Texte, sowie bisweilen auch Musiknotationen, wurden niedergeschrieben oder - falls bereits publiziert - beigelegt.

5 Das kann freilich auch dazu führen, dass gelegentlich Aufnahmen unterschiedlicher Sprachen eine Serie bilden, deren Gemeinsamkeit lediglich in der Person des Forschers liegt. Bisweilen kann es zudem sinnvoll sein, bereits früher edierte Aufnahmen nochmals in anderem, aber ebenfalls thematisch passenden bzw. erweiterten Zusammenhang zu veröffentlichen. Dies hätte für die Benutzer den Vorteil, dass alle Aufnahmen eines Genres, einer Sprache etc. in einer Serie vereinigt wären. Im Gegensatz zu im Rahmen von Feldforschung entstandenen Aufnahmen werden Archivaufnahmen hingegen eher nach inhaltlichen Aspekten gruppiert. 
lich dessen einfachere Auswertung) und erhöht die Benutzerfreundlichkeit.

2. Quellenkritische Aufbereitung der Protokolle: Nach der Zusammenstellung von Kurzinformationen zu Inhalt, Ort und Datum der Aufnahme sowie zur phonographierten Person (Name, Alter, Beruf, Herkunft) ist eine Ab- bzw. Neufassung von Orientierungs-Transkriptionen auf Basis heute üblicher Praxis vorzunehmen. Sie dienen lediglich dem besseren Verständnis und sollen daher - im Falle von Sprachaufnahmen bewusst keine detaillierten phonetischen Umschriften bieten. Die bereits vorhandenen Transkriptionen sind erneut durch Abhören der Tonaufnahmen zu überprüfen, da sie zumeist vor der eigentlichen Aufnahme gemacht wurden und daher gelegentlich vom tatsächlich Aufgenommenen abweichen können. Editorische Korrekturen/Ergänzungen (wie z.B. aktuelle geografische Bezeichnungen) oder auch im Protokoll notierte, aber letztendlich nicht aufgenommene Passagen werden mittels [ ], Versprecher/Wortwiederholungen o.ä. durch ( ) signalisiert; besonders bei nicht verschriftlichten oder aussterbenden Sprachen sowie Minderheitensprachen sind ggf. (Teil-)Übersetzungen (bei Liedern auch Incipits) sowie kurze Anmerkungen hilfreich.

3. Kontextualisierung mittels weiterer (schriftlicher) (Archiv-)Quellen: In der Regel enthalten die Beiträge im Kommentarteil zur Gesamtausgabe des PhA Angaben zur Entstehung, Interpretation und wissenschaftsgeschichtlichen Einordnung der Aufnahmen, zu deren Evaluierung im Rahmen der jeweiligen Disziplin sowie biografische Angaben zu den Feldforschern und Phonographierten. Die auf diese Weise erfolgte Kontextualisierung ist unabdingbar dafür, dass das Tondokument auch als wissenschaftliche Quelle bestehen kann. Dabei gilt: Die kommentierte Quellenedition soll die detaillierte wissenschaftliche Auswertung der Aufnahmen ermöglichen, jedoch nicht vorwegnehmen. Aufgrund der Heterogenität der Bestände handelt es sich bei der Herausgabe einer CD-Serie jedenfalls um ein interdisziplinäres Projekt, das sich nur im Team und mithilfe externer Kooperationen zielführend realisieren lässt. Der Kontakt mit Fachvertretern der entsprechenden Disziplinen sowie mit Angehörigen der jeweiligen, seinerzeit aufgenommenen Community ist daher unerlässlich. Als hilfreich bei der Kontextualisierung haben sich auch Recherchen etwa im Österreichischen Staatsarchiv, im Archiv der Universität Wien oder in wissenschaftlichen Nachlässen (v.a. hinsichtlich Korrespondenz der Feldforscher) sowie das Studium von zeitgenössischen Presseberichten erwiesen - insbesondere dann, wenn (womöglich mangelhaft ausgefüllte) Protokolle die einzige im $\mathrm{PhA}$ vorliegende Dokumentation ist und auch im Archiv der ÖAW keine weiteren Quellen vorhanden sind.

\section{Workflow II: Technische Aspekte ${ }^{6}$}

\subsection{Einführung}

Die Historischen Bestände 1899-1950 umfassen ca. 3200 sogenannte Phonogramme und ca. 800 Grammophonaufnahmen. ${ }^{7}$ Hinsichtlich der Tonqualität der Phonogramme stellen Lechleitner und Schüller (1999) 35 fest:

\begin{abstract}
„They suffer from heavy linear distortions, i.e. deviations from a linear frequency response, from non-linear distortions and from wow and flutter, speed variations caused by the uneven rotation of the mechanical recording devices. Mechanical rumble, rough recording surfaces, and low recording levels are the reasons for generally poor signal-to-noise ratios of most of the recordings. Additionally, due to imperfections of and damages to the recording surfaces of the original recording media, impulsive noises are found in the replay signals, which generally far exceed the signal levels.“
\end{abstract}

Die sieben Stationen, die generell bei der Edition historischer Tonaufnahmen zu durchlaufen sind, hat bereits Dietrich Schüller (1991) klar formuliert; darauf basieren auch jene sieben „Ebenen der Restaurierung“, die Wallaszkovits $^{8}$ folgendermaßen beschreibt:

6 Die folgenden Ausführungen beruhen größtenteils auf Interviews mit Kollegen am PhA (v. a. Franz Lechleitner, Johannes Spitzbart und Nadja Wallaszkovits). Als Standardliteratur zum Thema sei auf Schüller (1991), IASA-TC 03 (2005) und IASA-TC 04 (2009) sowie Casey und Gordon (2007), Copeland (2008) und Wallaszkovits (2009) verwiesen. Nicht zuletzt aufgrund des gänzlichen Fehlens von Quellennachweisen weniger empfehlenswert, wenn auch sehr praxisorientiert, ist Zander (2009).

7 Schüller (1999) 11: „Die Phonogrammsammlung lag ursprünglich in zweifacher Form vor: als vernickelte Kupfer-Matrizen (Negative) sowie als ,Archivplatten' (Positive) in Form von Wachsabgüssen bzw. galvanoplastisch erzeugten Metallplatten. Durch Kriegseinwirkung wurden die historischen Positive 1945 zerstört, die erhalten gebliebenen Matrizen ermöglichten jedoch Neuabgüsse, die 1962-1964 mittels Epoxidharz durchgeführt wurden. Diese dienen als Basis für die modernen Übertragungen. Die Grammophonsammlung war von einem Kriegsschaden nicht betroffen.“

Bei den mittels „Archivphonographen“ hergestellten Phonogrammen handelt es sich um Aufnahmen in Edison'scher Tiefenschrift auf Wachsplatte.

8 Wallaszkovits (2009) 600-03. 
1. „Die erste Ebene der Restaurierung beginnt mit der Frage nach dem Original bzw. mit der Wahl des zu übertragenden Tonträgers.“

2. Die zweite Ebene ist „der rein physischen Erhaltung des Originalträgers, bzw. der Spielbarmachung desselben" gewidmet.

3. Die dritte Ebene „umfasst die Wahl der Wiedergabegeräte, die zur sachgerechten Übertragung verwendet werden sollen“.

4. Die vierte „Ebene der unerlässlichen Restaurierungsschritte“ gilt der „Kompensation von intendierten Signalveränderungen, die bei der Aufnahme angewendet wurden, wie z.B. Entzerrung oder Rauschunterdrückung“.

5. „Die fünfte Ebene umfasst die Kompensation von möglichen Fehljustierungen bei der Aufnahmegerätschaft“, wie z.B. „den Ausgleich von Fehlern im Schneidwinkel bei Platten in Tiefenschrift".

6. „Die sechste Ebene betrifft die Kompensation von nicht intendierten Signalveränderungen, und bezeichnet in den meisten Fällen den Startpunkt der digitalen Signalbearbeitung“. Dazu zählen die Anwendung von Filtern „zur Kompensation von Nichtlinearitäten im Frequenzgang, verursacht z.B. durch Unzulänglichkeiten der Aufnahmegeräte (etwa Trichterresonanzen), das Entfernen von [...] Nadelgeräuschen, transienten Störgeräuschen etc.“.

7. Die siebte Ebene, oder „Re-Interpretation auf dem Tonmeister-Level“, erstreckt sich auf „sämtliche subjektive Bearbeitungsschritte, die der Bearbeitende hinzufügt, um das endgültige Ergebnis klanglich zu optimieren (subjektive Filterungen, Hinzufügen von Raumanteilen etc.)“.

Der Ansatz des PhA zielt nun darauf ab, „die Aufnahme unter bestmöglicher Anwendung der Ebenen 1-5 in der sechsten Ebene so aufzubereiten, dass die aufnahme- und wiedergabeseitigen Artefakte möglichst objektiv kompensiert werden“ - doch „sollten im Zweifelsfall Artefakte des Originals belassen und dokumentiert bzw. kommentiert" und „Artefakte des Restaurierungsvorganges [...] nicht in das Signal eingebracht werden“. ${ }^{9}$ Somit ergibt sich untenstehender Workflow, der die Praxis im PhA darlegt und sich in erster Linie auf Phonogramme, z.T. aber auch auf Wachszylinder und Grammophonplatten anwenden lässt.

9 Wallaszkovits (2009) 604.

\subsection{Reinigung}

Zunächst wird das Phonogramm einer Reinigung unterzogen: zuerst trocken (weicher Pinsel), dann nass (mit temperiertem Wasser und einem Netzmittel, z.B. H10, oder einfachem Spülmittel), wodurch die Oberflächenspannung des Wassers gebrochen und die Rillen auch in der Tiefe gesäubert werden; anschließend erfolgt die Trocknung an der Luft. ${ }^{10}$ Alternativ kann die Reinigung auch mit einer Record Cleaning Machine (aqua bidest./Netzmittel H10) erfolgen (z. B. Loricraft Record Cleaning Machine oder Keith Monks Record Cleaning Machine).

\subsection{Re-recording}

Unter Re-recording versteht man in diesem Zusammenhang die Übertragung von Aufnahmen auf mechanischen Tonträgern mittels Plattenspieler und Tonabnehmersystem. ${ }^{11}$ Dazu empfiehlt die International Association of Sound and Audiovisual Archives Folgendes:

„It is mandatory that transfers made from old to new archive formats be carried out without subjective alterations or 'improvements' such as de-noising, etc. It is essential that the full

10 Vgl. auch IASA-TC 04 (2009) 34-36.

11 Laut Nadja Wallaszkovits gäbe es zumindest zwei, derzeit aber weniger praktikable Alternativen:

1. optische Abtastung mittels Analogfotografie, Digitalisierung durch Scannen und Anwendung weiterer Bildanalyseverfahren. Zu den Vorteilen zählen die Erzeugung eines hochauflösenden Fotos auf stabilem Filmmaterial (zugleich eine Sicherheitskopie des Originals), das Fehlen mechanischer Einwirkung beim Re-recording und die Eignung für den Einsatz bei gesprungenen/gebrochenen Platten sowie die weitreichenden grafischen Bearbeitungsmöglichkeiten des digitalen Abbildes der Rille. Von Nachteil ist jedoch v.a. die Tatsache, dass aufgrund der Körnigkeit des Filmmaterials, Unschärfe etc. die Signalqualität derzeit noch schlechter ist als bei Nadelabtastung. Fazit: Vielversprechende Ergebnisse für lateral geschnittene Aufnahmen auf Platte (Seitenschrift), doch liegen keinerlei Erfahrungen mit der Abtastung der Tiefenschrift von Phonogrammen vor. Für gekrümmte Medien (z. B. Zylinder) ist diese Methode nicht geeignet.

2. Laserabtastung (noch nicht ausgereift): Die unter dem Begriff $3 D$ Scanning bekannte Methode wurde vom Lawrence Berkeley National Laboratory und der Library of Congress entwickelt. Das Medium wird mit einem polychromatischen Laser-Array abgetastet, wodurch sich ein dreidimensionales Profil der Rille erstellen lässt. Die Analyse- und Konvertierungssoftware erlaubt ebenso umfangreiche grafische Bearbeitungsmöglichkeiten des digitalen Abbildes der Rille. Fazit: Ideal für bereits geschädigte oder gebrochene Platten und Zylinder, als nachteilig erweisen sich jedoch die - im Vergleich zur mechanischen Abtastung - geringere erzielbare Audioqualität sowie die lange Bearbeitungszeit. 
dynamic range and frequency response of the original is transferred.

It is important to understand that the intended signal is only part of a given sound document. The unintended and undesirable artefacts (noise, distortions) are also part of the sound document, either caused by limited historical recording technology, or subsequently added to the original signal by mishandling (e.g. clicks) or by poor storage. Both have to be preserved with utmost accuracy [...].

Better transfers of the unintended parts of a sound document [... make the future removal of these artefacts by digital signal processing easier“..$^{12}$

Die Vorgangsweise des $\mathrm{PhA}$ lässt sich nun so zusammenfassen: ${ }^{13}$

„Die Übertragung erfolgt mittels elektromagnetischen StereoPickups unter Anwendung aller Sorgfaltsmaßnahmen des modernen Re-recording (Einsatz hochwertiger Übertragungsgeräte, Zentrierung an den Trägern und Nadelwahl). Die linear verstärkten Signale des Stereo-Pickups werden als Rohübertragungen gespeichert [...] und dienen als Ausgang für die weiteren Bearbeitungen. Das Mono-Signal der vertikal modulierten Phonogramme wird durch Differenz-, das der lateral modulierten Grammophonplatten durch Summenbildung der Signale des StereoPickups erzielt.“

Das PhA verfügt über folgendes Set-up für das Re-recording:

1. Abspielgerät: Diapason, ein modifizierter TechnicsPlattenspieler (Technics SFTG 172-01, „Diapason Archive Turntable“) mit variabler Umdrehungszahl (ca. 15-120 rpm, revolutions per minute), regulierbarem Anti-Skating sowie höhenverstellbarem Tonarm (SME Series III) - wichtig aufgrund der unterschiedlichen Höhe historischer Tonträger - mit Öldämpfung und Tonabnehmersystem Shure M44-7.

2. Vorverstärker (AHE Audio Heritage Equipment Preamplifier PA-02, entwickelt von Franz Lechleitner): Durch die Abspielung mittels Stereosystem wird die vertikale Modulation der Phonogramme durch Differenzbildung der beiden Kanäle hergestellt.

Vor dem Re-recording sind folgende vier Arbeitsschritte vorzunehmen:

1. Einstellung der Geschwindigkeit: Die empfohlene Aufnahmegeschwindigkeit für Sprache bzw. Musik betrug in der Regel zwischen 50 und $70 \mathrm{rpm}$, wobei $50 \mathrm{rpm}$ etwa einer Aufnahmedauer von zwei Minuten entsprachen. Diese Angaben waren jedenfalls auch in den Protokollen zu notieren, sind zumeist korrekt und so-

12 IASA-TC $03(2005) 7 \mathrm{f}$

13 Schüller (1999) 11, vgl. IASA-TC 04 (2009) 36-39. mit die Ausgangsbasis für die Einstellung der Geschwindigkeit, die aber dennoch gelegentlich auditiv zu korrigieren ist.

2. Auswahl der Nadel: Für die Übertragung von Phonogrammen hat sich eine bi-radiale (quasi-elliptische) Nadel bewährt (Abmessungen: $229 \mu \mathrm{m}$ x $61 \mu \mathrm{m}$, Gewicht: $50 \mathrm{mN}),{ }^{14}$ wobei sicherheitshalber auch stets eine Dimension darüber/darunter zu testen und auditiv zu bewerten ist. Eine Rille kann beispielsweise in mittlerer Höhe mechanische Schäden aufweisen, die durch entsprechende Auswahl der Nadel beim Auslesen des Signals umgangen werden können.

3. Zentrierung der Platte: Nachdem die Epoxidharz-Abgüsse der Phonogramme über kein normiertes Mittelloch verfügen, ist die perfekte Zentrierung des Phonogramms (bei laufender Platte und aufgelegter Nadel) von großer Bedeutung, da ansonsten Tonhöhenschwankungen auftreten, die sich nachträglich nicht entfernen lassen.

4. Anti-Skating: Durch entsprechende Einstellung des Anti-Skating wird verhindert, dass die Nadel beim Abspielen an die Rilleninnenkante gedrückt wird und bei seichten Rillen dadurch herausspringt (Skating). Dies passiert bei Phonogrammen bei Rillenverschnitt (hervorgerufen durch Aus- und erneutes Einschalten während des Aufnahmevorgangs) häufig. ${ }^{15} \mathrm{Da}$ das Skating sowohl von der Geschwindigkeit als auch vom jeweiligen Trägermaterial abhängig ist, muss das Anti-Skating beim Re-recording von Phonogrammen unterschiedlicher Geschwindigkeit bzw. vor dem Wechsel zu anderen Tonträgern neu eingestellt werden.Die wichtigsten technischen Parameter (Tonabnehmer + Nadel, Geschwindigkeit, Angaben zur Einstellung des Vorverstärkers) werden in einem Übertragungsprotokoll notiert und zusammen mit weiteren Details als Preservation Metadata in der internen Archiv-Datenbank abgespeichert. ${ }^{16}$

14 Das Ziel ist, das stärkste, unverzerrte Signal zu erreichen. Für (kommerzielle) Platten mit seichter Rille ist eine Nadel mit kegelstumpfartiger Spitze (truncated stylus) ideal.

15 Abhilfe könnte hier ein Re-recording mit halber Geschwindigkeit bringen, vgl. IASA-TC 04 (2009) 39, doch wird durch die digitale Korrektur im Anschluss das Rauschen verstärkt.

16 Die Preservation Metadata, oft als Untergruppe der Administrative Metadata gesehen (vgl. IASA-TC 04 (2009) 18f.) umfassen somit folgende Informationen: „[T]he original carrier, its format and state of preservation; replay equipment for the original carrier and its parameters; the digital resolution, file format information, and all equipment used; the operators involved in the process; checksum - the digital signature that permits authentication of the file; details of the secondary information sources“, vgl. IASA-TC 03 (2005) 10. 


\subsection{Digitalisierung}

Die Digitalisierung erfolgt im Wesentlichen auf Basis der von der UNESCO als „best practice“ empfohlenen Guidelines on the Production and Preservation of Digital Audio Objects. ${ }^{17}$

Zielformat bei der Digitalisierung (Archivstandard: 24 bit/96 kHz; bei zu erwartender, aufwendiger Restaurierung alternativ auch $24 \mathrm{bit} / 192 \mathrm{kHz}$ ) ist das lineare (also nicht datenreduzierte) Waveform Audio File Format (WAVE), mittlerweile das gängige Standardformat. Die dafür verwendeten Geräte sind ein Vorverstärker (s.o.) und ein externer, professioneller A/D-Wandler (z.B. Lake People ADDAC F 446), wobei ein Audio-Editor (wie etwa WaveLab) als Software dient.

\subsection{Schnitt}

Ausgangspunkt sind die Rohübertragungen, jene, die aus mechanischen Gründen (Kratzer, Höhenschlag bei seichten Rillen etc.) nicht in einem kontinuierlichen Durchgang gemacht werden können, müssen vor der Signalverbesserung zunächst geschnitten werden. Angesichts der Zielvorgabe, so viel wie möglich vom Inhalt zu übertragen, gestaltet sich dieser Prozess bei stark beschädigten Phonogrammen aus zwei Gründen als arbeitsintensiv:

1. Die Nadel überspringt bei beschädigter Oberfläche unkontrolliert Rillen, wodurch mehrere Versionen unterschiedlichen Inhalts bzw. unterschiedlicher Länge entstehen.

2. Durch mehrmaliges Übertragen erhält man verschiedene Versionen bzw. Fragmente, aus denen eine Aufnahme zusammengesetzt werden muss.

Der auf diese Weise entstandene Rohschnitt wird als digitale Archivkopie unter einer eigenen Signatur gespeichert und in der internen Archiv-Datenbank erfasst, um a) eine erneute Signalverbesserung zu einem zukünftigen Zeitpunkt vornehmen und auf diese Weise von der Weiterentwicklung der Technik profitieren zu können, ${ }^{18}$ und b) eine Referenzversion zu haben, die eine Überprüfung der signalverbessernden Eingriffe ermöglicht.

\subsection{Signalverbesserung}

Das Ergebnis dieses Arbeitsvorgangs, der nach dem Prinzip „So viel wie nötig, so wenig wie möglich“ abläuft und auch psychoakustische Aspekte berücksichtigt, ${ }^{19}$ ist eine Datei, deren Signal - im Gegensatz zu jenem von Rohübertragung und Rohschnitt - modifiziert wurde. Diese signalverbesserte Version wird als „Master“ bezeichnet und bildet die Basis für die Edition. Als guter Überblick sei einleitend die $\mathrm{Zu}$ sammenfassung aus den „Editionsprinzipien“ zitiert: $^{20}$

\begin{abstract}
„Auf den Audio-CDs werden im wesentlichen sogenannte flat transfers publiziert, bei denen das im Frequenzgang unbehandelte, also linear verstärkte Signal lediglich von groben Oberflächenfehlern (,Knacksern') befreit und mit einer, jeder Aufnahme individuell angepaßten, Bandbegrenzung versehen wird. Die Entfernung der groben Oberflächenfehler ermöglicht einen höheren Signalpegel auf der CD, während eine hohe obere Grenzfrequenz das Nutzsignal garantiert unbeeinflußt läßt. Dem durch das Rauschen irritierten Hörer bleibt eine individuelle Höhenbegrenzung unbenommen [...]. Um den Hörer auf die historische Tonqualität vorzubereiten, werden Einlaufrillen ein-, Auslaufrillen ausgeblendet. Bei abrupt beginnenden bzw. endenden Aufnahmen wird Rauschen aus dem Kontext zum Ein- und Ausblenden benutzt. Eine weitere Signalbearbeitung, etwa Entrauschen, wird generell nicht vorgenommen.“
\end{abstract}

Die Signalverbesserung wird mittels einer integrierten Workstation für Audiorestaurierung in nachstehender Reihenfolge durchgeführt (basierend auf den im PhA verwendeten Plug-ins, den AudioCube-VPIs):

1. De-Clicker: Entfernen von punktuellen, starken transienten Störgeräuschen („Knacksern“),

2. DeCrackler: Entfernen von „Knistern“ über die gesamte Aufnahme hinweg,

3. RepairFilter: Hoch- und Tiefpassfilter, bei Bedarf notch filter (Kerbfilter zum Entfernen von schmalbandigen Störgeräuschen), Live Spektralanalyse,

4. Waveform Restorer: dient dem manuellen Entfernen von punktuellen „Knacksern“ (im Gegensatz zu DeClicker und DeCrackler, die - einmal eingestellt - auf die gesamte Aufnahme angewendet werden),

5. SpectraPolator: erlaubt eine frequenzspezifische Auswahl und somit die selektive Beseitigung von Störgeräuschen auf Basis einer Spektralanalyse (anders als der Waveform Restorer, der auf die Wellenform also auf das gesamte Spektrum - abzielt),

19 Vgl. dazu Wallaszkovits (2009) 608.

20 Schüller (1999) $11 \mathrm{f}$.
17 IASA-TC 04 (2009).

18 Vgl. IASA-TC 03 (2005) 7.

\author{
-
}


6. SpectralDeHiss Expert II: ermöglicht das Entrauschen auf Basis eines individuell analysierten Rauschprofils der jeweiligen Aufnahme,

7. analogEQ: finales Entrauschen und Entrumpeln der Aufnahme durch Pegelabsenkung außerhalb des Nutzsignalfrequenzspektrums (Equalizer).

Die Stufen 3-6 kommen nur im Bedarfsfall zum Einsatz. Besonderer Wert wird dabei darauf gelegt, dass alle Methoden entsprechend maßvoll eingesetzt werden, um möglichst keine hörbaren Artefakte der Restaurierungsschritte in den Signalweg einzubringen. ${ }^{21}$

Abschließend muss darauf hingewiesen werden, dass es für den Einsatz von Restaurierungs-Tools „bisher noch keine allgemein angewandten Empfehlungen als ethische und ästhetische Entscheidungshilfen" gibt. ${ }^{22}$ Es ist folglich immer eine individuelle Einschätzung vorzunehmen. ${ }^{23}$ Wallaszkovits (2009) 607 präsentiert dazu hilfreiche Überlegungen, die als „Grundgerüst“ dienen mögen:

1. Digitale Restaurierung indiziert:

a) bei nicht intendierten Aufnahmefehlern, dazu zählt z.B. „ein stärkeres Rauschen, das aufgrund von Gleichlaufschwankungen ein regelmäßiges Muster aufweist“, hervorgerufen durch eine Platte, die „bei der Aufnahme nicht ganz plan aufgelegt war",

b) bei der sich ändernden Klangfarbe des Rauschens, die ja „rein medial bedingt“ ist, ${ }^{24}$

21 In jenen Fällen, wo der Cube-Tec DeClicker und DeCrackler suboptimale Ergebnisse liefern, kommen - alternativ oder zusätzlich der CEDAR DC-1 De-Clicker und der CR-1 De-Crackler zum Einsatz.

22 Wallaszkovits (2009) 609.

23 Laut Wallaszkovits (2009) 606f. ist die Klangqualität prinzipiell „beeinflusst durch das Aufnahmegerät, den verwendeten Trichter, das verwendete Diaphragma, die gewählte Geschwindigkeit, die Dynamik und Position der Schallquelle, sowie die Geschicklichkeit des Forschers“, wobei „[h]örbare Artefakte des Aufnahmevorganges [...] Ein- und Ausschaltgeräusche, die zu spät angesetzte Einlaufrille (Anfang der Aufnahme fehlt), Resonanzen des Aufnahmeapparates, Rillenverschnitt“ etc. darstellen.

24 Wallaszkovits erläutert dies so (ebd.): „Die Drehzahl ist beim Phonogramm zwar konstant, aber die Abtastgeschwindigkeit der Rille wird nach innen hin langsamer. Da aber die auftretenden Reibungskräfte geschwindigkeitsabhängig sind, ändert sich die Klangfarbe des Rauschens. [...] Sollen nun mehrere Phonogrammplatten zu einer Aufnahme zusammengeschnitten werden, was der Intention des Aufnehmenden entspricht, jedoch durch die zwei Minuten Begrenzung des Mediums nicht möglich war, so kommt es zu unterschiedlicher Klangfärbung zwischen dem Ende einer Platte und dem Anfang der nächsten Platte. Diese Klangfärbung ist rein medial bedingt, daher könnte eine entsprechende Filterung zur Anpassung in Erwägung gezogen werden.“ c) bei nicht intendierten Artefakten, „die durch den modernen Reproduktionsvorgang und die Wiedergabe entstehen“, wie z.B. „eine verminderte Signalqualität durch Körnigkeit und Qualität des Abgusses, Resonanzen des Wiedergabesystems, nichtlineare Verzerrungen durch Geometrie der Abtastnadel und des Tonarmes, Verzerrungen durch Probleme beim Zentrieren der Phonogramme, Rillensprünge und dadurch erfolgte Abtastung in Teilen",

2. Digitale Restaurierung nicht indiziert: Bei Ein- und Ausschaltgeräuschen infolge einer Aufnahme in Teilen. Werden diese „bei der Restaurierung entfernt, um eine Aufnahme im Ganzen zu simulieren [...], werden dem Hörer wichtige Informationen vorenthalten und eine wissenschaftliche Auswertung führt $\mathrm{zu}$ falschen Ergebnissen“. ${ }^{25}$

\subsection{Mastering}

Das Audio-CD-Mastering wird wieder im WaveLab durchgeführt und umfasst folgende Arbeitsschritte:

1. Angleichen der Lautstärke,

2. Anordnen der Tracks in der für die Edition gewählten Reihenfolge, Arrangieren der Tracks (also das Zusammenfassen von zwei oder mehreren zusammengehörigen Phonogrammen zu einer Tracknummer),

3. Konvertieren in das Audio-CD-Format (16 bit/44,1 kHz) unter Anwendung von dithering und noise shaping,

4. Fade-in/Fade-out der einzelnen Aufnahmen,

5. Kontrolle der Audio-CD-Parameter und Erstellen/ Brennen der Master-CD für die Produktion.

\section{Abschließende Bemerkung}

Zusammenfassend lässt sich sagen, dass die vorgestellten Workflows zu inhaltlichen und technischen Aspekten der Edition historischer Tonaufnahmen zwar auf der Praxis des Wiener PhA basieren, doch in vielen Bereichen von

25 Zum besseren Verständnis hier die Erklärung von Wallaszkovits (ebd.): „Aufgrund der Kürze des Mediums wurde der Aufnahmeapparat nach jeder Strophe abgeschaltet, damit Melodie und Text der folgenden Strophe von der aufzunehmenden Person noch einmal memoriert werden konnte und keine ungenutzten Leerstellen am Phonogramm entstanden. [...] Da zwischen den Aufnahmeteilen einige Zeit vergehen konnte, merkten sich Sänger oft nicht genau die Tonhöhe, in der sie das Lied begonnen hatten, und so kommt es vor, dass mitten im Lied mit unterschiedlicher Tonhöhe weitergesungen wurde.“ 
allgemeiner Gültigkeit sind und somit - neben der nach wie vor wichtigen persönlichen Einschulung vor Ort -auch anderen Institutionen Orientierung und einen ersten Überblick bieten können.

\section{Literaturverzeichnis}

Casey, Mike; Bruce Gordon (2007): Sound Directions: Best Practices for Audio Preservation. [Bloomington]: Indiana University; [Cambridge, MA]: Harvard University. Verfügbar unter http://www.dli b.indiana.edu/projects/sounddirections/papersPresent/index. shtml.

Copeland, Peter (2008): Manual of Analogue Sound Restoration Techniques. London: The British Library. Verfügbar unter http://ww w.bl.uk/help/manual-of-analogue-audio-restoration-techni ques.

IASA-TC 03 (2005): IASA-TC 03 = The Safeguarding of the Audio Heritage: Ethics, Principles and Preservation Strategy. Version 3, hg. v. Dietrich Schüller. [Budapest]: IASA (IASA Technical Committee: Standards, Recommended Practices and Strategies; IASA-TC 03). Verfügbar unter http://www.iasa-web.org/tc03/eth ics-principles-preservation-strategy.

IASA-TC 04 (2009): IASA-TC 04 = Guidelines on the Production and Preservation of Digital Audio Objects. 2. Ausg., hg. v. Kevin Bradley. [Auckland Park]: IASA (IASA Technical Committee: Standards, Recommended Practices and Strategies; IASA-TC 04). Verfügbar unter http://www.iasa-web.org/tc04/audio-preserva tion.

Lechleitner, Franz; Schüller, Dietrich (1999): On the re-recording of the edition. In: The First Expeditions 1901 to Croatia, Brazil, and the Isle of Lesbos, hg. v. Dietrich Schüller. Wien: VÖAW (Tondokumente aus dem Phonogrammarchiv der Österreichischen Akademie der Wissenschaften: Gesamtausgabe der Historischen Bestände 1899-1950, Series 1, OEAW PHA CD 7), 35-37.

Liebl, Christian (2015): Zur Edition historischer Tonaufnahmen: Vorarbeiten für die Erschließung und Kontextualisierung unver- öffentlichter Bestände des Phonogrammarchivs der Österreichischen Akademie der Wissenschaften. Masterthese des Universitätslehrgangs Library and Information Studies MSc an der Universität Wien in Kooperation mit der Österreichischen Nationalbibliothek.

Schüller, Dietrich (1991): The Ethics of Preservation, Restoration, and the Re-Issues of Historical Sound Recordings. In: Journal of the Audio Engineering Society, 39 (12), 1014-17.

Schüller, Dietrich (1999): Editionsprinzipien. In: Stimmporträts, hg. v. Dietrich Schüller. Wien: VÖAW (Tondokumente aus dem Phonogrammarchiv der Österreichischen Akademie der Wissenschaften: Gesamtausgabe der Historischen Bestände 1899-1950, Serie 2, OEAW PHA CD 8), 11-15.

Wallaszkovits, Nadja (2009): Digitale Restaurierung historischer Tonaufnahmen: Eine Gratwanderung zwischen Authentizität und Manipulation (Digital Restoration of Historical Audio Recordings: A Balancing Act between Authenticity and Manipulation). In: Proceedings of the 25. Tonmeistertagung: VDT International Audio Convention, 13th-16th November 2008, Congress Center Leipzig. Leipzig: Bildungswerk des Verbandes Deutscher Tonmeister, 598-610.

Zander, Horst (2009): PC-gestützte Restaurierung von Audiosignalen. Berlin: Schiele \& Schön.

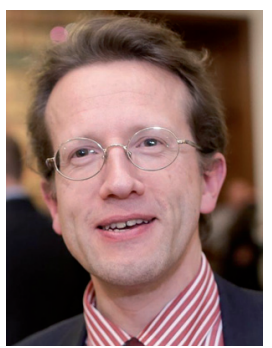

Christian Liebl

Österreichische Akademie der

Wissenschaften

Liebiggasse 5

A-1010 Wien

Österreich

Christian.Liebl@oeaw.ac.at 